\title{
Intracultural Variation in the Knowledge of Medicinal Plants in an Urban-Rural Community in the Atlantic Forest from Northeastern Brazil
}

\author{
Cecília de Fátima Castelo Branco Rangel de Almeida, ${ }^{1}$ Marcelo Alves Ramos, ${ }^{1}$ \\ Rafael Ricardo Vasconcelos Silva, ${ }^{1}$ Joabe Gomes de Melo, ${ }^{1}$ Maria Franco Trindade Medeiros, ${ }^{1}$ \\ Thiago Antonio de Sousa Araújo, ${ }^{1}$ Alyson Luiz Santos de Almeida, ${ }^{1}$ \\ Elba Lúcia Cavalcanti de Amorim, ${ }^{2}$ Rômulo Romeu da Nóbrega Alves, ${ }^{3}$ \\ and Ulysses Paulino de Albuquerque ${ }^{1}$
}
${ }^{1}$ Laboratory of Applied Ethnobotany, Department of Biology, Federal Rural University of Pernambuco, Avenida Dom Manoel de Medeiros s/n, Dois Irmãos, 52171-900 Recife, PE, Brazil
${ }^{2}$ Natural Products Laboratory, Pharmacy Department, Federal University of Pernambuco, 50670-901 Recife, PE, Brazil
${ }^{3}$ Ethnozoology, Conservation and Biodiversity Research Group, Departamento de Biologia, Universidade Estadual da Paraíba, 58109-753 Campina Grande, PB, Brazil

Correspondence should be addressed to Cecília de Fátima Castelo Branco Rangel de Almeida, ccastelobranco@yahoo.com and Ulysses Paulino de Albuquerque, upa677@hotmail.com

Received 23 June 2011; Revised 17 August 2011; Accepted 23 August 2011

Academic Editor: Ana H. Ladio

Copyright (๑) 2012 Cecília de Fátima Castelo Branco Rangel de Almeida et al. This is an open access article distributed under the Creative Commons Attribution License, which permits unrestricted use, distribution, and reproduction in any medium, provided the original work is properly cited.

\begin{abstract}
This study assessed the intracultural knowledge of the use of medicinal plants in an urban-rural community in an Atlantic forest fragment in northeastern Brazil. We examined the importance of native and exotic species and the effects of gender and age on that knowledge. We also compared data obtained from different groups of informants (local experts and general community). We conducted 194 interviews between June 2007 and January 2008, using the freelist technique and semistructured forms to collect ethnobotanical data. Information obtained from the community was compared with that from six local experts who participated in a survey in 2003. From a total of 209 ethnospecies, exotic and herbaceous plants presented higher richness. With respect to the number of citations, women and older informants were shown to know a higher number of medicinal plants. Comparing knowledge of local experts with that of the general community, we noted that experts know a similar wealth of plant families and therapeutic indications, but the community knows a greater species richness. These results indicate that local experts may provide useful information for studies that search for a quick diagnosis of the knowledge of a given community.
\end{abstract}

\section{Introduction}

In Brazil, the Atlantic Forest is one of the most biologically diverse ecosystems, responsible for harboring a large number of endemic species $[1,2]$. It extends from Rio Grande do Norte to Rio Grande do Sul [3] and, given its location in the coastal area, is currently under strong pressure from real estate speculation. In addition, there are the pressures generated by timber extraction, the cycles of sugar cane, coffee, and gold, and, more recently, the expansion of farming and forestry with exotic species.
Human populations living in the surrounding areas of the Atlantic Forest play an important role in its exploitation as they often rely on forest resources for their subsistence and extract biological resources from it on a daily basis [4]. Understanding how these people use such resources is a task of great current interest, which may contribute to the discovery of products of economic interest and to the conservation of biological resources.

Thus, ethnobotanical studies can contribute to assessing how local knowledge is distributed among members of a community and the relationship between that knowledge 
and the increase of exotic species in the local repertoire of medicinal plants [5-7].

Common knowledge about plant resources, especially medicinal ones, is highly dynamic and subject to several influences, may vary according to gender, age, education level, income, roles that individuals play within the family, skills, and abilities [8-11], and may represent key elements of the knowledge of the diversity and richness of species [12].

Different social patterns have been reported to impact the knowledge of medicinal plants, emphasizing the need of studies that address such questions. For instance, Almeida et al. [13] did not observe any differences between the knowledge of men and women, whereas age and income were correlated with the number of citations for a given plant and its indication, suggesting that older people with a higher income had greater knowledge about such plant resources.

Thus, the goal of this study was to assess the intracultural knowledge of the use of medicinal plants in an urban-rural community in an Atlantic forest fragment in northeastern Brazil in order to document the importance of native and exotic species within the group of plants mentioned and the effects of gender and age on the knowledge of medicinal plants and to compare the quality of information gathered from different groups of informants (local experts versus general community).

\section{Materials and Methods}

2.1. Study Area. The study was conducted at Igarassu, located in the microregion of Itamaracá and the mesoregion of Recife, in Pernambuco state $\left(7^{\circ} 50^{\prime} 20^{\prime \prime} \mathrm{S}\right.$ and $35^{\circ} 00^{\prime} 10^{\prime \prime} \mathrm{W}$; $20 \mathrm{~m}$ a.s.1.), $30 \mathrm{~km}$ from the state capital [14-16]. The climate is tropical, hot, and humid, with autumn/winter rains (according to the classification of Köeppen). The average annual temperature is $27^{\circ} \mathrm{C}$, and the average annual rainfall is approximately $2,000 \mathrm{~mm}$ [14-16]. The municipality has a total area of $304.2 \mathrm{~km}^{2}$, with a population of 72,990 people (219.9 inhabitants $/ \mathrm{km}^{2}$ ), $74.9 \%$ of which live in urban areas [14].

The predominant vegetation is composed of remnants of Atlantic forest, secondary forests, mangroves, palm trees, and areas of commercial and subsistence agriculture. There are ecological reserves in the city, such as the São José Plant Forest, with tall, dense vegetation, located on Transcanavieira Highway (PE-41) and with an area of 323.30 ha [17].

The community studied is known as "Três Ladeiras" and is located on the lands of the "São José Plant," a sugar refinery. The "São José Plant" is surrounded by Atlantic forest fragments belonging to an ecological reserve [18]. The forest is part of the conservation area of the Botafogo River basin, in accordance with state law no. 9860, which since August 12, 1986, has been aimed at protecting the landscape, soil, and river basin [19]. The fragments occupy a total area of 210 ha [18]. The community lies $30 \mathrm{~km}$ north of the county seat and is located at the back of a large hill, whose extension contains three elevations that give the community its name. The district has 1,794 inhabitants, of which 1,077 live in urban areas and 687 in rural ones [20].
Most men from the community work at the plant although the number of people employed by the refinery oscillates during the year, increasing and decreasing according to season and periods of land preparation, planting, and harvesting [15]. It is not unusual to find among the residents of the community families with small fields that provide nutritional and/or economic support during periods when there is no work at the plant [15]. There is no sanitation, medical care takes place in a health clinic for minor health problems, and disease control is provided by health workers through weekly home visits. Patients who require extra care are relocated to hospitals in the county seat of Igarassu.

2.2. Data Collection. Ethnobotanical data were obtained through the freelist technique, followed by semistructured interviews [21]. The interviews were conducted with the senior member of the family, over 18 years old, present on the visit of the interviewer. Initially, we obtained a Term of Informed Consent from those willing to participate in the study in accordance with the legal and ethical aspects of Resolution 196/96 from the Ethics and Research Committee [22].

Because the community had a large number of residents, we sampled $51 \%$ of all households and conducted 194 interviews (140 women and 54 men) between June 2007 and January 2008. The age of informants ranged from 18 to 93 years. For the interviews, one main question was asked: "What medicinal plants do you know?". In a second event, we gathered information on each species mentioned, the part of the plant used, the method of preparation, its indication and contraindication, as well as socioeconomic data from informants, such as gender, age, family income, and number of residents in the household. Ages were grouped into five different groups, ranging from 18 to over 68 years.

We used the data obtained in this study and in the work of Gazzaneo et al. [15] to compare the information obtained from the general community and local experts, respectively. The latter study was conducted in the same community in 2003 and was attended by six informants identified as "local experts," given their more detailed knowledge on the use of medicinal plants [23]. This group of informants was composed of three men and three women, with ages ranging from 51 to 102 years. The data sampling performed by Gazzaneo et al. [15] was intentionally nonrandom and assumed that local experts provide more specific, high-quality information about medicinal plants. To select this group of informants, the authors used the "snowball" method [24]. Data were collected using semistructured interviews that gathered information related to the knowledge of medicinal plants.

\footnotetext{
2.3. Species Categorization and Indications Mentioned by Informants. All plants mentioned during interviews were identified and classified as either native or exotic species according to their biogeographical origin. We considered native species those endemic to the study region and also native to South America. Exotic species were considered to be those of extracontinental origin, cultivated in the region, and widely distributed, such as tropical invasive and cosmopolitan species.
} 
To calculate the relative importance of species, all indications mentioned by the informants were grouped into 18 disease categories, according to the classification from the World Health Organization [25]: digestive, respiratory, gynecological/urinary, circulatory, nervous, sensory, motor, puerperium, cutaneous, scarring, poisoning, neoplasia, hematopoietic, nutritional, infectious/parasitic, sexual inappetence and antiabortion, and postpartum. Diseases not categorized by the aforementioned system were grouped into the category "undefined ailments and pains" by virtue of their symptoms and signs of multiple origins [26].

All species mentioned by informants, excluding those commercialized, were collected, identified, and deposited in the herbaria of Professor Geraldo Mariz (UFP), at the Federal University of Pernambuco, Professor Dárdano de Andrade Lima (IPA), at the Agricultural Research Company, and Professor Sérgio Tavares (HST), at the Federal Rural University of Pernambuco.

2.4. Data Analysis. We calculated the value of relative importance (RI) for all species [27] with the following formula: $\mathrm{RI}=\mathrm{NBS}+\mathrm{NP}$, where NBS is the number of body systems treated by a particular species (NBSS) divided by the total number of body systems treated by the most versatile species (NBSVS) and NP is the number of attributed properties of a particular species (NPS) divided by the total number of properties attributed to the most versatile species (NPVS).

The chi-squared adherence test was used to check for differences between the following factors: number of native versus exotic plants and number of plants observed in each life form (herb, shrub, and tree). We also compared the richness of exclusive species between different age groups, richness of families, total number of mentioned species, and number of exclusive species between local experts and the general community.

We used the Kruskal-Wallis nonparametric test to test for differences in the richness of ethnospecies and mentioned indications between men and women and between each age group and to check for differences between the relative importance of species mentioned by local experts and the general community.

We used Williams' G-test to compare the proportion of the number of native and exotic species (exclusive or not) mentioned by local experts and the general community.

The Spearman correlation test was applied to check for the relationship between the number of ethnospecies and the number of mentioned indications according to the age of the informants and to check for a relationship between relative importance (RI) of species mentioned by the general community and the RI calculated for local experts.

All statistical analyses were performed using the statistical package BioEstat 5.0 [28].

\section{Results}

3.1. Richness of Medicinal Plants Mentioned by Informants. In total, 209 ethno-species were mentioned during interviews; 151 were identified to the species level and 21 to the genus level only (Table 1). The plants were distributed in 74

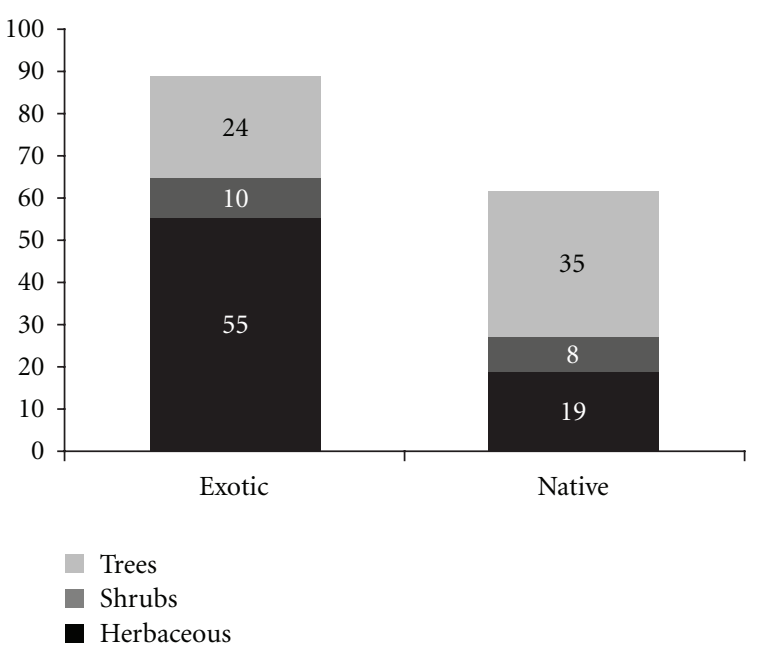

FIGURE 1: Origin and life form of the medicinal species mentioned in the community of Três Ladeiras, Igarassu, Pernambuco state, northeastern Brazil.

families, and most families (66\%) were represented by up to two species. The most represented families were Lamiaceae (10 spp.); Caesalpiniaceae and Curcubitaceae (8 spp.); Asteraceae, Euphorbiaceae, and Mimosaceae (5 spp.).

With respect to the origin of the identified species, we observed that 89 were exotic and 62 were native (Figure 1), and the difference was statistically significant $\left(\chi^{2}=4.8\right.$; $P<0.05$ ). That result indicates that informants knew more exotic plants that could be used for medicinal purposes. With respect to the life form of plants, there was a predominance of herbs (74), followed by trees (59) and shrubs (18) (Figure 1), but we only observed statistical differences when we compared the richness of shrubs with that of herbs $\left(\chi^{2}=\right.$ 34.01; $P<0.0001)$ and trees $\left(\chi^{2}=21.83 ; P<0.0001\right)$. The number of herbs and trees was not significantly different $\left(\chi^{2}=1.7 ; P=0.23\right)$, indicating that the richness of herbs and trees was similar in the pool of plants mentioned by informants. However, when considering the distribution of species according to their origin, we observed a different pattern: for exotic plants, there was a higher number of herbaceous plants compared to the other two life forms (shrubs: $\chi^{2}=31.15, P<0.0001$; trees: $\chi^{2}=12.16, P=$ $0.005)$, whereas, for native plants, there was a higher number of trees (herbs: $\chi^{2}=4.7, P=0.02$; shrubs: $\chi^{2}=16.95$, $P<0.0001)$.

The most mentioned species were Schinus terebinthifolius Raddi (aroeira-219 citations), Alpinia zerumbet (Pers.) B. L. Burtt \& R. M. Sm. (colônia-190 citations), Pithecellobium cochliocarpum (Gomez) Macbr. (babatenon-183), Plectranthus amboinicus (Lour.) Spreng (hortelã graúda-155), Mentha piperita L. (hortelã miúda-141), and Cymbopogon citratus (DC.) Stapf (capim santo-133) (Table 1). Except for $S$. terebinthifolius and $P$. cochliocarpum, all these plants are exotic, emphasizing the importance of exotic plants to the knowledge of medicinal plants in the region.

3.2. Influence of Gender and Age on the Knowledge of Medicinal Plants. There were significant differences in the knowledge 
TABle 1: Medicinal plants mentioned in the community of Três Ladeiras, Igarassu, Pernambuco state, Brazil.

\begin{tabular}{|c|c|c|c|c|c|c|}
\hline Family/scientific name & Vernacular name & Habit & Origin & Citation number & RI $2005^{*}$ & RI 2008 \\
\hline \multicolumn{7}{|l|}{ Acanthaceae } \\
\hline Justicia pectoralis Jacq. & Chambá & Herbs & Native & 21 & 0.33 & 0.37 \\
\hline Justicia sp. & Anador & - & - & 25 & - & 1.00 \\
\hline Graptophyllum pictum (L.) Griff. & Melacilina & Herbs & Exotic & 15 & 0.67 & 0.94 \\
\hline \multicolumn{7}{|l|}{ Amaranthaceae } \\
\hline Alternanthera brasiliana (L.) Kuntze. & Novalgina & Herbs & Exotic & 3 & - & 0.35 \\
\hline Pfaffia glomerata (Spreng.) Pederson & Acônico/Acônito & Herbs & Exotic & 17 & 0.67 & 0.27 \\
\hline \multicolumn{7}{|l|}{ Anacardiaceae } \\
\hline Anacardium occidentale L. & Cajueiro roxo & Tree & Native & 113 & 0.33 & 1.24 \\
\hline Mangifera indica L. & Manga & Tree & Exotic & 11 & 0.83 & 0.62 \\
\hline Schinus terebinthifolius Raddi & Aroeira & Tree & Native & 219 & 1.17 & 1.60 \\
\hline Spondias purpurea L. & Siriguela & Tree & Native & 3 & - & 0.27 \\
\hline \multicolumn{7}{|l|}{ Annonaceae } \\
\hline Annona montana Macfad. & Aticum & Tree & Native & 13 & 0.83 & 0.61 \\
\hline Annona muricata L. & Graviola & Tree & Exotic & 4 & - & 0.30 \\
\hline Annona squamosa $\mathrm{L}$. & Pinha & Tree & Exotic & 1 & - & 0.12 \\
\hline \multicolumn{7}{|l|}{ Apiaceae } \\
\hline Daucus carota L. & Cenoura & Herbs & Exotic & 1 & - & 0.12 \\
\hline Foeniculum vulgare Mill. & Endro & Herbs & Exotic & 12 & - & 0.74 \\
\hline Pimpinella anisum L. & Erva doce & Herbs & Exotic & 45 & 0.33 & 1.09 \\
\hline \multicolumn{7}{|l|}{ Apocynaceae } \\
\hline Catharanthus roseus L. G. Don. & Boa noite branca & Herbs & Exotic & 3 & - & 0.27 \\
\hline Hancornia speciosa Gomes & Mangaba & Tree & Native & 1 & 0.17 & 0.12 \\
\hline \multicolumn{7}{|l|}{ Arecaceae } \\
\hline Acrocomia aculeata (Jacq.) Lodd. ex Mart. & Macaiba & Tree & Native & 30 & 0.17 & 0.76 \\
\hline Cocos nucifera L. & Coco amarelo & Tree & Exotic & 7 & 0.17 & 0.39 \\
\hline Elaeis guineensis Jacq. & Dendezeiro & Tree & Exotic & 2 & 0.17 & 0.24 \\
\hline Syagrus sp. & Coco catolé & Tree & Native & 6 & 0.50 & 0.42 \\
\hline \multicolumn{7}{|l|}{ Asteraceae } \\
\hline Acanthospermum hispidum DC. & Espinho de cigano & Herbs & Exotic & 48 & 0.67 & 0.76 \\
\hline Conyza bonariensis (L.) Cronq. & Rabo de raposa & Herbs & Exotic & 3 & 0.33 & 0.12 \\
\hline Helianthus annuus L. & Girassol & Herbs & Native & 10 & 0.17 & 0.12 \\
\hline Matricaria chamomilla L. & Camomila & Herbs & Exotic & 7 & - & 0.12 \\
\hline Pluchea sp. & Mar de cravo & Herbs & - & 1 & - & 0.12 \\
\hline $\begin{array}{l}\text { Gymnanthemum amygdalinum (Delile) } \\
\text { Sch.Bip. ex Walp. }\end{array}$ & Alcachofra & Herbs & Exotic & 50 & 0.33 & 0.83 \\
\hline Asteraceae 1 & Carqueja & - & - & 2 & - & 0.12 \\
\hline \multicolumn{7}{|l|}{ Begoniaceae } \\
\hline Begonia reniformis Dryand. & Capeba & Shrub & Native & 3 & 0.17 & 0.24 \\
\hline \multicolumn{7}{|l|}{ Bignoniaceae } \\
\hline $\begin{array}{l}\text { Handroanthus impetiginosus (Mart. ex DC.) } \\
\text { Mattos }\end{array}$ & Pau daico roxo & Tree & Native & 8 & 0.67 & 0.54 \\
\hline \multicolumn{7}{|l|}{ Bombacaceae } \\
\hline Chorisia sp. & Barriguda & Tree & Native & 1 & - & 0.12 \\
\hline \multicolumn{7}{|l|}{ Boraginaceae } \\
\hline Heliotropium angiospermum Murray & Crista de galo & Herbs & Exotic & 1 & - & 0.12 \\
\hline Heliotropium indicum L. & Fedegoso & Herbs & Exotic & 3 & 0.50 & 0.35 \\
\hline
\end{tabular}


Table 1: Continued.

\begin{tabular}{|c|c|c|c|c|c|c|}
\hline Family/scientific name & Vernacular name & Habit & Origin & Citation number & RI $2005^{*}$ & RI 2008 \\
\hline \multicolumn{7}{|l|}{ Brassicaceae } \\
\hline Nasturtium officinale R. Br. & Agrião & Herbs & Exotic & 48 & 0.83 & 0.53 \\
\hline \multicolumn{7}{|l|}{ Bromeliaceae } \\
\hline Ananas comosus (L.) Merr. & Abacaxi & Herbs & Native & 14 & - & 0.39 \\
\hline Tillandsia usneoides (L.) L. & Salambaia & Herbs & Native & 2 & 0.17 & 0.24 \\
\hline \multicolumn{7}{|l|}{ Burseraceae } \\
\hline Protium heptaphyllum (Aubl.) Marchand & Amescla & Tree & Native & 1 & 1.00 & 0.12 \\
\hline \multicolumn{7}{|l|}{ Cactaceae } \\
\hline Cereus jamacaru DC. & Cardeiro & Tree & Native & 5 & - & 0.12 \\
\hline \multicolumn{7}{|l|}{ Caesalpiniaceae } \\
\hline Bauhinia forficata Link & Pata de vaca & Tree & Native & 9 & - & 0.30 \\
\hline Caesalpinia echinata Lam. & Pau brasil & Tree & Native & 1 & - & 0.12 \\
\hline Caesalpinia ferrea Mart. ex Tul. & Jucá & Tree & Native & 11 & 0.50 & 0.27 \\
\hline Copaifera sp. & Pau dóleo & Tree & - & 1 & 0.17 & 0.12 \\
\hline Hymenaea martiana Hayne & Jatobá & Tree & Native & 27 & 1.33 & 1.20 \\
\hline Senna alata (L.) Roxb. & Café beirão & Shrub & Exotic & 1 & - & 0.12 \\
\hline Senna occidentalis (L.) Link. & Manjirioba/Mata pasto & Herbs & Exotic & 24 & 0.17 & 0.76 \\
\hline Poincianella pyramidalis (Tul.) L. P. Queiroz & Catingueira & Tree & Native & 1 & - & 0.12 \\
\hline \multicolumn{7}{|l|}{ Capparaceae } \\
\hline Cleome spinosa Jacq. & Mussambê & Shrub & Exotic & 11 & 0.17 & 0.30 \\
\hline \multicolumn{7}{|l|}{ Caprifoliaceae } \\
\hline Sambucus nigra L. & Flor de sabugo/Sabugueiro & Tree & Exotic & 33 & 0.50 & 0.57 \\
\hline \multicolumn{7}{|l|}{ Caricaceae } \\
\hline Carica papaya L. & Mamão & Tree & Native & 7 & 0.33 & 0.46 \\
\hline \multicolumn{7}{|l|}{ Caryophyllaceae } \\
\hline Dianthus caryophyllus L. & Cravo branco & Herbs & Exotic & 4 & - & 0.27 \\
\hline \multicolumn{7}{|l|}{ Cecropiaceae } \\
\hline Cecropia palmata Willd. & Embauba branca & Tree & Native & 6 & 0.33 & 0.42 \\
\hline \multicolumn{7}{|l|}{ Chenopodiaceae } \\
\hline Beta vulgaris L. & Beterraba & Herbs & Exotic & 8 & - & 0.34 \\
\hline Chenopodium ambrosioides $\mathrm{L}$. & Mastruz/Mentruz & Herbs & Exotic & 85 & 0.67 & 0.68 \\
\hline \multicolumn{7}{|l|}{ Chrysobalanaceae } \\
\hline Licania sp. & Oiti/Oiticica & - & - & 2 & - & 0.24 \\
\hline Chrysobalanaceae 1 & Oiticoró & - & - & 1 & - & 0.12 \\
\hline \multicolumn{7}{|l|}{ Clusiaceae } \\
\hline Vismia guianensis (Aubl.) Pers. & Lacre & Tree & Native & 12 & 0.33 & 0.42 \\
\hline \multicolumn{7}{|l|}{ Combretaceae } \\
\hline Buchenavia sp. & Imbiriba & - & - & 4 & 0.33 & 0.15 \\
\hline Terminalia catappa $\mathrm{L}$. & Coração de negro & Tree & Exotic & 2 & - & 0.58 \\
\hline \multicolumn{7}{|l|}{ Convolvulaceae } \\
\hline Ipomoea asarifolia (Ders.) R. et Sch & Salsa & Herbs & Exotic & 1 & 0.17 & 0.15 \\
\hline Operculina alata (Ham.) Urb. & Batata de purga & Herbs & Native & 1 & 0.50 & 0.12 \\
\hline Convolvulaceae 1 & Acanfó/Acafú & - & - & 10 & 0.33 & 0.34 \\
\hline Convolvulaceae 2 & Sassá & - & - & 1 & - & 0.12 \\
\hline \multicolumn{7}{|l|}{ Crassulaceae } \\
\hline Kalanchoe laciniata (L.) DC. & Corona branca & Herbs & Exotic & 17 & - & 0.81 \\
\hline Kalanchoe sp. & Corona roxa & - & - & 6 & - & 0.51 \\
\hline
\end{tabular}


Table 1: Continued.

\begin{tabular}{|c|c|c|c|c|c|c|}
\hline Family/scientific name & Vernacular name & Habit & Origin & Citation number & RI $2005^{*}$ & RI 2008 \\
\hline \multicolumn{7}{|l|}{ Cucurbitaceae } \\
\hline Citrullus vulgaris Schard. & Melância & Herbs & Exotic & 1 & - & 0.12 \\
\hline Cucumis anguria $\mathrm{L}$. & Maxixe & Herbs & Exotic & 1 & - & 0.12 \\
\hline Cucumis melo L. & Melão & Herbs & Exotic & 1 & - & 0.12 \\
\hline Cucumis sativus L. & Pepino & Herbs & Exotic & 2 & - & 0.24 \\
\hline Curcubita pepo L. & Jerimum & Herbs & Exotic & 8 & - & 0.24 \\
\hline Luffa operculata L. Cong. & Cabacinha & Herbs & Native & 1 & 0.17 & 0.12 \\
\hline Momordica charantia L. & Melão de são caetano & Herbs & Exotic & 1 & - & 0.12 \\
\hline Sechium edule (Jacq.) Sw. & Chuchu & Herbs & Exotic & 20 & 0.17 & 0.27 \\
\hline \multicolumn{7}{|l|}{ Equisetaceae } \\
\hline Equisetum sp. & Cavalinha & Herbs & - & 1 & - & 0.12 \\
\hline \multicolumn{7}{|l|}{ Euphorbiaceae } \\
\hline Cnidosculus urens (L.) Arthur & Urtiga branca & Herbs & Native & 25 & 0.50 & 0.68 \\
\hline Euphorbia tirucalli L. & Aveloz & Shrub & Exotic & 1 & - & 0.12 \\
\hline Jatropha gossypiifolia L. & Pinhão roxo & Shrub & Exotic & 5 & - & 0.35 \\
\hline Jatropha mollissima (Pohl.) Baill. & Pinhão branco & Shrub & Native & 2 & - & 0.24 \\
\hline Manihot esculenta Crantz & Macacheira/Roça & Herbs & Native & 1 & - & 0.12 \\
\hline Phyllanthus niruri L. & Quebra pedra & Herbs & Exotic & 13 & 0.17 & 0.46 \\
\hline Ricinus communis $\mathrm{L}$. & Carrapateira/Mamona & Shrub & Exotic & 5 & - & 0.27 \\
\hline \multicolumn{7}{|l|}{ Fabaceae } \\
\hline Bowdichia virgilioides Kunth & Sucupira & Tree & Native & 5 & 0.50 & 0.39 \\
\hline Vicia faba L. & Fava & Herbs & Exotic & 1 & - & 0.12 \\
\hline Zornia diphylla (L.) Pers. & Urinana & Herbs & Native & 3 & 0.17 & 0.15 \\
\hline \multicolumn{7}{|l|}{ Flacourtiaceae } \\
\hline Flacourtiaceae 1 & Imbira branca & - & - & 2 & - & 0.12 \\
\hline \multicolumn{7}{|l|}{ Heliconiaceae } \\
\hline Heliconia psittacorum L. f. & Paquivira & Herbs & Native & 1 & 一 & 0.12 \\
\hline \multicolumn{7}{|l|}{ Illiaceae } \\
\hline Illicium verum Hook. f. & Anil estrelado & Tree & Exotic & 9 & - & 0.73 \\
\hline \multicolumn{7}{|l|}{ Iridaceae } \\
\hline Crocus sp. & Açafrão & Herbs & - & 1 & 0.33 & 0.12 \\
\hline Eleutherine bulbosa (Mill.) Urb. & Alho do mato & Herbs & Native & 3 & 0.67 & 0.15 \\
\hline \multicolumn{7}{|l|}{ Lamiaceae } \\
\hline Aeollanthus suaveolens Mart. ex Spreng. & Macassá & Herbs & Exotic & 32 & 1.33 & 1.13 \\
\hline Mentha piperita L. & Hortelã miúda & Herbs & Exotic & 141 & 0.83 & 2.00 \\
\hline Mentha pulegium L. & Hortelã pastilha/H. vick & Herbs & Exotic & 34 & 0.17 & 1.07 \\
\hline Ocimum basilicum L. & $\begin{array}{l}\text { Manjericão/Manjericão são } \\
\text { josé }\end{array}$ & Herbs & Exotic & 50 & 0.50 & 1.16 \\
\hline $\begin{array}{l}\text { Ocimum basilicum var. minimum (Willd.) } \\
\text { Benth. }\end{array}$ & Manjericão miúdo & Herbs & Exotic & 5 & 0.17 & 0.35 \\
\hline Ocimum campechianum Mill. & Alfavaca de caboclo & Herbs & Native & 14 & - & 0.69 \\
\hline Ocimum gratissimum $\mathrm{L}$. & $\begin{array}{c}\text { Louro falso/L. caseiro/ } \\
\text { Hortelã fernando/H. são } \\
\text { severino }\end{array}$ & Herbs & Exotic & 4 & 0.17 & 0.24 \\
\hline Plectranthus amboinicus (Lour.) Spreng. & $\begin{array}{l}\text { Hortelã graúda/H. } \\
\text { gorda/H. bahia }\end{array}$ & Herbs & Exotic & 155 & 0.83 & 1.22 \\
\hline Plectranthus barbatus Andrews & $\begin{array}{l}\text { Boldo caseiro/Boldo } \\
\text { falso/Hortelã caboclo }\end{array}$ & Herbs & Exotic & 17 & 0.50 & 0.84 \\
\hline Rosmarinus officinalis $\mathrm{L}$. & Alecrim & Herbs & Exotic & 11 & 0.17 & 0.96 \\
\hline Lamiaceae 1 & Alfazema & Herbs & - & 1 & - & 0.12 \\
\hline Lamiaceae 2 & Alfazema de caboclo & Herbs & - & 1 & - & 0.12 \\
\hline Lamiaceae 3 & Veiga morta & Herbs & - & 53 & 0.50 & 0.84 \\
\hline
\end{tabular}


TABle 1: Continued.

\begin{tabular}{|c|c|c|c|c|c|c|}
\hline Family/scientific name & Vernacular name & Habit & Origin & Citation number & RI $2005^{*}$ & RI 2008 \\
\hline \multicolumn{7}{|l|}{ Lauraceae } \\
\hline Nectandra cuspidata Ness \& Mart. & Canela & Tree & Native & 71 & - & 0.95 \\
\hline Persea americana Mill. & Abacate & Tree & Exotic & 15 & 0.83 & 0.62 \\
\hline \multicolumn{7}{|l|}{ Liliaceae } \\
\hline Allium cepa L. & Cebola & Herbs & Exotic & 12 & 0.67 & 0.34 \\
\hline Allium sativum $\mathrm{L}$. & Alho & Herbs & Exotic & 19 & 0.33 & 0.56 \\
\hline Aloe vera (L.) Berm.f. & Baborsa/Erva babosa & Herbs & Exotic & 32 & 0.83 & 1.13 \\
\hline \multicolumn{7}{|l|}{ Loranthaceae } \\
\hline Loranthaceae 1 & Cipó estanca sangue & - & - & 1 & 0.33 & 0.12 \\
\hline \multicolumn{7}{|l|}{ Malpighiaceae } \\
\hline Byrsonima sericea DC. & Murici & Tree & Native & 1 & - & 0.12 \\
\hline Malpighia glabra L. & Acerola & Tree & Exotic & 10 & - & 0.51 \\
\hline \multicolumn{7}{|l|}{ Malvaceae } \\
\hline Gossypium barbadense L. & Algodão & Shrub & Exotic & 2 & 0.50 & 0.20 \\
\hline Uerna lobata L. & Malva rosa & Herbs & Native & 11 & 0.33 & 0.51 \\
\hline \multicolumn{7}{|l|}{ Meliaceae } \\
\hline Cedrela odorata L. & Cedro & Tree & Native & 1 & - & 0.12 \\
\hline \multicolumn{7}{|l|}{ Mimosaceae } \\
\hline Acacia sp. & Espinheiro & Tree & - & 3 & - & 0.12 \\
\hline Anadenanthera colubrina (Vell.) Brenan. & Angico & Tree & Native & 3 & - & 0.24 \\
\hline Inga bahiensis Benth. & Inga porco & Tree & Native & 1 & - & 0.12 \\
\hline Mimosa tenuiflora (Willd.) Poir. & Jurema preta & Tree & Native & 2 & - & 0.12 \\
\hline Piptadenia stipulacea (Benth.) Ducke. & Jurema branca & Tree & Native & 1 & - & 0.12 \\
\hline $\begin{array}{l}\text { Pithecellobium cochliocarpum (Gomez) } \\
\text { Macbr. }\end{array}$ & Babatenom & Tree & Native & 183 & 1.83 & 1.14 \\
\hline Pithecellobium saman var. acutifolium Benth. & Budão de velho & Tree & Native & 2 & - & 0.24 \\
\hline \multicolumn{7}{|l|}{ Monimiaceae } \\
\hline Peumus boldus Mol. & Boldo do chile & Herbs & Exotic & 44 & - & 0.73 \\
\hline \multicolumn{7}{|l|}{ Moraceae } \\
\hline Artocarpus communis J.R. Forst. \& G. Forst. & Fruta pão & Tree & Exotic & 3 & - & 0.35 \\
\hline Artocarpus integrifolia L. f. & Jaca & Tree & Exotic & 2 & - & 0.24 \\
\hline Dorstenia sp. & Conta erva & - & - & 1 & 0.83 & 0.12 \\
\hline \multicolumn{7}{|l|}{ Musaceae } \\
\hline Musa paradisiaca $\mathrm{L}$. & Bananeira & Tree & Exotic & 19 & 0.33 & 0.73 \\
\hline \multicolumn{7}{|l|}{ Myrtaceae } \\
\hline Eucalyptus citriodora Hook. & Eucalipto & Tree & Exotic & 7 & 0.17 & 0.24 \\
\hline Eugenia uniflora L. & Pitanga & Tree & Native & 58 & 0.17 & 0.59 \\
\hline Psidium guajava L. & Goiaba & Tree & Native & 51 & 0.17 & 0.24 \\
\hline Psidium guineense Sw. & Araça & Shrub & Native & 5 & 0.17 & 0.12 \\
\hline $\begin{array}{l}\text { Syzygium aromaticum (L.) Merr. \& L.M. } \\
\text { Perry }\end{array}$ & Cravo do reino & Tree & Exotic & 5 & - & 0.59 \\
\hline Syrygium jambolanum (Lam.) DC. & Azeitona preta & Tree & Exotic & 14 & 0.17 & 0.69 \\
\hline \multicolumn{7}{|l|}{ Nyctaginaceae } \\
\hline Boerhavia diffusa L. & Pega pinto & Herbs & Exotic & 22 & 1.17 & 0.83 \\
\hline Guapira sp. & João mole & Tree & - & 3 & - & 0.24 \\
\hline
\end{tabular}


Table 1: Continued.

\begin{tabular}{|c|c|c|c|c|c|c|}
\hline Family/scientific name & Vernacular name & Habit & Origin & Citation number & RI 2005* & RI 2008 \\
\hline \multicolumn{7}{|l|}{ Olacaceae } \\
\hline Ximenia americana $\mathrm{L}$. & Ameixa & Tree & Native & 1 & 0.17 & 0.12 \\
\hline \multicolumn{7}{|l|}{ Oxalidaceae } \\
\hline Averrhoa carambola $\mathrm{L}$. & Carambola & Tree & Exotic & 17 & 0.83 & 0.30 \\
\hline \multicolumn{7}{|l|}{ Papaveraceae } \\
\hline Argemone mexicana L. & Cardo santo & Herbs & Exotic & 7 & 0.50 & 0.24 \\
\hline \multicolumn{7}{|l|}{ Passifloraceae } \\
\hline Passiflora edulis Sims. & Maracujá & Shrub & Native & 12 & 0.17 & 0.49 \\
\hline \multicolumn{7}{|l|}{ Pedaliaceae } \\
\hline Sesamum orientale L. & Gergelim preto/Gigilim & Herbs & Exotic & 4 & 0.17 & 0.24 \\
\hline \multicolumn{7}{|l|}{ Phytolacaceae } \\
\hline Petiveria alliacea $\mathrm{L}$. & Timpi & Herbs & Native & 7 & 0.67 & 0.54 \\
\hline \multicolumn{7}{|l|}{ Piperaceae } \\
\hline Peperomia pellucida H.B.K. & Lingua de sapo & Herbs & Native & 5 & - & 0.39 \\
\hline Piper nigrum L. & Pimenta do reino & Shrub & Exotic & 2 & 一 & 0.24 \\
\hline \multicolumn{7}{|l|}{ Poaceae } \\
\hline Cymbopogon citratus (DC.) Stapf & Capim santo & Herbs & Exotic & 133 & 0.50 & 1.47 \\
\hline Imperata brasiliensis Trin. & Sapé & Herbs & Native & 5 & - & 0.15 \\
\hline Phalaris canariensis $\mathrm{L}$. & Alpiste & Herbs & Exotic & 4 & 0.17 & 0.24 \\
\hline Zea mays L. & Milho & Herbs & Exotic & 2 & - & 0.12 \\
\hline \multicolumn{7}{|l|}{ Polygalaceae } \\
\hline Polygala sp. & Esquentado & - & 一 & 1 & 一 & 0.12 \\
\hline \multicolumn{7}{|l|}{ Punicaceae } \\
\hline Punica granatum L. & Romã & Tree & Exotic & 14 & 一 & 0.49 \\
\hline \multicolumn{7}{|l|}{ Rhamnaceae } \\
\hline Zizyphus joazeiro Mart. & Juá & Tree & Native & 22 & 0.83 & 0.76 \\
\hline \multicolumn{7}{|l|}{ Rosaceae } \\
\hline Pyrus malus L. & Maçã & Tree & Exotic & 1 & - & 0.12 \\
\hline Rosa sp.1 & Rosa amélia & - & - & 1 & - & 0.12 \\
\hline Rosa sp.2 & Rosa branca & 一 & 一 & 7 & 0.33 & 0.62 \\
\hline \multicolumn{7}{|l|}{ Rubiaceae } \\
\hline Borreria verticillata L. G. Mey. & Vassoura de botão & Herbs & Exotic & 47 & 1.17 & 1.51 \\
\hline Genipa americana L. & Jenipapo & Tree & Native & 53 & - & 0.64 \\
\hline $\begin{array}{l}\text { Uncaria tomentosa (Willd. ex Roem. \& } \\
\text { Schult.) DC. }\end{array}$ & Unha de gato & Tree & Native & 1 & - & 0.12 \\
\hline \multicolumn{7}{|l|}{ Rutaceae } \\
\hline Citrus limetta Risso & Lima & Tree & Exotic & 1 & - & 0.12 \\
\hline Citrus limonia Osbeck & Limão & Tree & Exotic & 14 & - & 0.57 \\
\hline Citrus sinensis (L.) Osbeck & Laranja & Tree & Exotic & 44 & 0.83 & 0.93 \\
\hline Ruta graveolens L. & Arruda & Herbs & Exotic & 75 & 0.83 & 1.08 \\
\hline \multicolumn{7}{|l|}{ Sapindaceae } \\
\hline Cardiospermum halicacabum L. & Cipó de vaqueiro & Shrub & Native & 10 & 0.33 & 0.24 \\
\hline Cupania sp. & Cabotam & - & - & 1 & 0.33 & 0.12 \\
\hline Serjania sp. & Cipó cururu & 一 & 一 & 2 & 一 & 0.12 \\
\hline \multicolumn{7}{|l|}{ Sapotaceae } \\
\hline Achras zapota L. & Sapoti & Tree & Exotic & 1 & - & 0.12 \\
\hline $\begin{array}{l}\text { Sideroxylon obtusifolium (Roem. \& Schult.) } \\
\text { T.D. Penn. }\end{array}$ & Quixaba & Tree & Native & 27 & 一 & 1.14 \\
\hline
\end{tabular}


TABle 1: Continued.

\begin{tabular}{|c|c|c|c|c|c|c|}
\hline Family/scientific name & Vernacular name & Habit & Origin & Citation number & RI $2005^{*}$ & RI 2008 \\
\hline \multicolumn{7}{|l|}{ Smilacaceae } \\
\hline Smilax rotundifolia $\mathrm{L}$. & Cipó japecanga & Herbs & Native & 6 & 0.17 & 0.12 \\
\hline \multicolumn{7}{|l|}{ Solanaceae } \\
\hline Capsicum frutescens $\mathrm{L}$. & Pimenta & Shrub & Native & 3 & - & 0.24 \\
\hline Nicotiana tabacum L. & Fumo & Herbs & Native & 1 & - & 0.12 \\
\hline Solanum americanum Mill. & Avamoura/Erva moura & Herbs & Exotic & 5 & 0.17 & 0.30 \\
\hline Solanum paniculatum L. & Jurubeba & Shrub & Exotic & 4 & 1.00 & 0.35 \\
\hline \multicolumn{7}{|l|}{ Sterculiaceae } \\
\hline Guazuma ulmifolia L. & Mutamba & Tree & Native & 7 & - & 0.24 \\
\hline \multicolumn{7}{|l|}{ Theacaea } \\
\hline Camellia sinensis (L.) Kuntze & Chá preto & Shrub & Exotic & 4 & - & 0.20 \\
\hline \multicolumn{7}{|l|}{ Turneracea } \\
\hline Turnera ulmifolia $\mathrm{L}$. & Xanana & Herbs & Exotic & 1 & 0.17 & 0.12 \\
\hline \multicolumn{7}{|l|}{ Verbenaceae } \\
\hline Lantana camara L. & Chumbinho & Shrub & Native & 5 & 0.33 & 0.19 \\
\hline Lippia alba (Mill.) N.E.Br. & Cidreira/Erva cidreira & Herbs & Exotic & 94 & 0.67 & 1.86 \\
\hline Stachytarpheta elatior Schrad. ex Schult & Mocotó & Herbs & Native & 4 & 0.67 & 0.12 \\
\hline Vitex agnus-castus $\mathrm{L}$. & Liamba & Tree & Exotic & 2 & 0.17 & 0.24 \\
\hline \multicolumn{7}{|l|}{ Violaceae } \\
\hline Hybanthus sp. & Pepaconha & - & - & 7 & - & 0.51 \\
\hline \multicolumn{7}{|l|}{ Vitaceae } \\
\hline Cissus verticillata (L.) Nicolson \& C.E. Jarvis & Insulina & Shrub & Native & 5 & 0.17 & 0.12 \\
\hline Leea sp. & Café & - & - & 3 & - & 0.27 \\
\hline Vitis vinifera $\mathrm{L}$. & Uva & Shrub & Exotic & 1 & - & 0.12 \\
\hline \multicolumn{7}{|l|}{ Zingiberaceae } \\
\hline $\begin{array}{l}\text { Alpinia zerumbet (Pers.) Burt. ex R. M. } \\
\text { Smith }\end{array}$ & Colônia/Colonha & Herbs & Exotic & 190 & 1.67 & 0.91 \\
\hline Costus sp. & Cana de macaco & - & - & 27 & - & 0.84 \\
\hline Zingiber officinalis Rosc. & Gengibre vermelho & Herbs & Exotic & 1 & - & 0.12 \\
\hline \multicolumn{7}{|l|}{ Unidentified } \\
\hline Unidentified 1 & Abre caminho & - & - & 2 & - & 0.12 \\
\hline Unidentified 2 & Açafroa & - & - & 1 & - & \\
\hline Unidentified 3 & Boca torta & - & - & 1 & - & 0.12 \\
\hline Unidentified 4 & Bugre & - & - & 2 & - & 0.12 \\
\hline Unidentified 5 & Cafofa & - & - & 1 & - & 0.12 \\
\hline Unidentified 6 & Canela de viado & - & - & 1 & - & 0.12 \\
\hline Unidentified 7 & Chumbinho branco & - & - & 1 & - & 0.12 \\
\hline Unidentified 8 & Cipó de boi & - & - & 2 & - & 0.24 \\
\hline Unidentified 9 & Imbira vermelha & - & - & 2 & - & 0.12 \\
\hline Unidentified 10 & Malicia branca & - & - & 2 & - & 0.24 \\
\hline Unidentified 11 & Malicia boi/M. fina & - & - & 4 & - & 0.15 \\
\hline Unidentified 12 & Malva ferro & - & - & 1 & - & 0.12 \\
\hline Unidentified 13 & Moça & - & - & 1 & - & 0.12 \\
\hline Unidentified 14 & Pé de galinha/Papo de peru & - & - & 1 & - & 0.12 \\
\hline Unidentified 15 & Pega rapaz & - & - & 1 & - & 0.12 \\
\hline Unidentified 16 & Perpetua branca & - & - & 1 & - & 0.12 \\
\hline
\end{tabular}


TABle 1: Continued.

\begin{tabular}{|c|c|c|c|c|c|c|}
\hline Family/scientific name & Vernacular name & Habit & Origin & Citation number & RI $2005^{*}$ & RI 2008 \\
\hline Unidentified 17 & Piripiri & - & - & 1 & - & 0.12 \\
\hline Unidentified 18 & Piriquiti & - & - & 1 & - & 0.12 \\
\hline Unidentified 19 & Quebra faca & - & - & 5 & - & 0.27 \\
\hline Unidentified 20 & Quentão & - & - & 1 & - & 0.12 \\
\hline Unidentified 21 & Rasteira & - & - & 1 & - & 0.12 \\
\hline Unidentified 22 & Rasteirinho & - & - & 1 & - & 0.12 \\
\hline Unidentified 23 & Salsa caroba & - & - & 1 & - & 0.12 \\
\hline Unidentified 24 & Sete casco & - & - & 1 & - & 0.12 \\
\hline Unidentified 25 & Tatajuba & - & - & 1 & - & 0.12 \\
\hline
\end{tabular}

* (-) denotes the absence of the species on the list of Gazzaneo et al. [15].

RI 2005: relative importance calculated from information given by local experts; RI 2008: relative Importance calculated from information given by the general community.

of medicinal plants according to gender, with women knowing a higher richness of ethno-species $(H=117.29$; $P=0.0006)$ and indications $(H=134 ; P=0.0003)$. They mentioned a total of 166 ethno-species, with a mean number of $13.33 \pm 7.84$ citations per person, and a total of 146 indications, with a mean of $10.16 \pm 5.73$ citations per person, whereas men mentioned 136 ethno-species and 93 indications, with a mean of $9.95 \pm 7.05$ and $7.51 \pm 4.9$ citations per person, respectively.

The number of plants and indications mentioned by each informant correlated with their age ( $r s=0.33, P<0.0001$; rs $=0.37, P<0.0001$, resp.). However, when we compared the average number of ethnospecies and indications in each age group, we observed different patterns (Table 2). The number of known plants only varied in informants from the 18-28-year-old age group, suggesting that the richness of known plants was smaller in younger participants, which may reflect the limited experience and contact of young informants with plant resources from the region. Although informants aged 49-58 years old had greater knowledge of medicinal plants in the region, they were only significantly different from younger informants (Table 2). With respect to the number of indications mentioned in each age group, we observed a similar pattern to the previous one, with younger informants (18-28 yrs.) knowing a smaller variety of indications. However, the knowledge of informants was significantly higher for the age groups 49-58 years old and older than in other age groups.

When we analyzed the influence of gender and age on the distribution of knowledge, we observed a few patterns that often differed from the data presented by the general community. Younger women (18-28 yrs.) also had less knowledge of the richness of medicinal species, while, in other age groups, knowledge was homogeneous. For women, we observed the formation of two groups regarding the number of indications: one group consisting of the three younger groups, with a lower number of indications, and the other consisting of older age groups, with a higher number of indications.

For men, knowledge of plant richness and indications showed a different pattern. The knowledge of informants in the 18-48-year-old age groups did not present any statistical

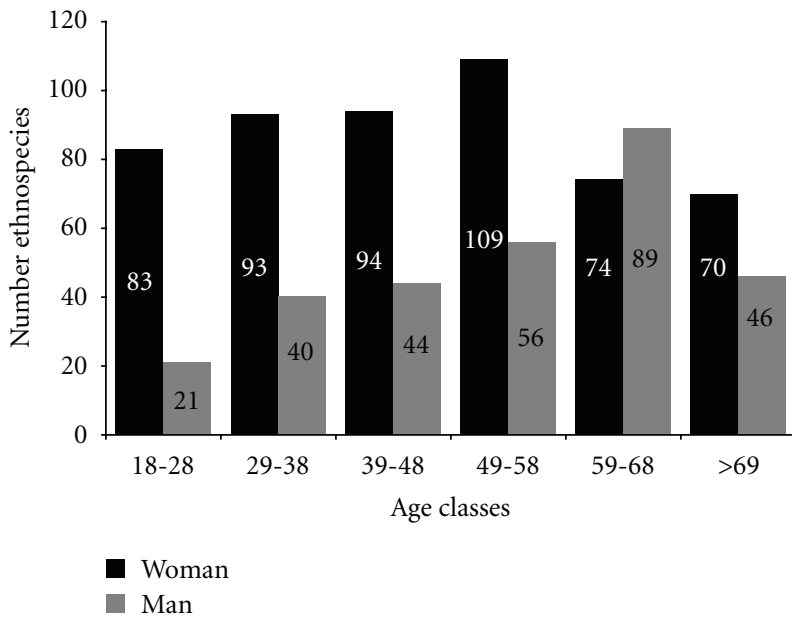

FIgure 2: Distribution of the knowledge of medicinal plants between men and women in the community of Três Ladeiras, Igarassu, Pernambuco state, northeastern Brazil.

differences. That difference only appeared in the age groups 49-58 years old and older, indicating an increase in the number of species known and the variety of indications occurring only in older age groups, whereas for women it was also observed in younger age groups.

We observed a continuous increase in the number of ethno-species mentioned with an increase in age, for both men and women, when total plant richness was considered (Figure 2), up to the age group with the highest richness of plants mentioned. In subsequent age groups, plant richness started to decrease. Among women, the 49-58-yearold age group had the greatest knowledge of plants (109 ethno-species), whereas for men the greatest knowledge of plants was observed in an older class (59-68 yrs.). These results indicate that, in the community of Three Hills, the commitment of women to family care compels them to know, from an early age, a large number of plants with medicinal purposes.

Following the age groups with a higher number of plants, there was a decrease in plant richness in older groups, 


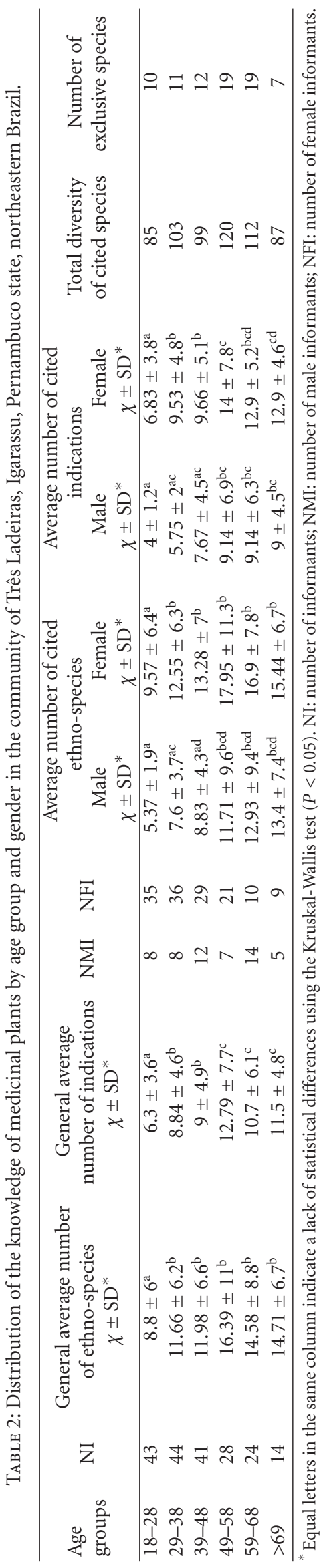


possibly related to memory loss, which is common among older people. We did not observe the same pattern when the mean number of plants mentioned by informants was analyzed, as previously noted (Table 2 ). In this case, people still mentioned a high number of citations, even in the oldest age group ( $>69$ yrs.).

Exclusive species were mentioned in all age groups (Table 2), with a total richness of 78 exclusive species distributed in the six age groups. The 49-58- and 59-68year-old age groups stand out for their higher richness, although there were no statistical differences in the number of exclusive species between age groups $\left(\chi^{2}=9.38 ; P=\right.$ 0.09 ), indicating that, in every age group, informants had a repertoire of exclusive plants that was not shared by people from other age groups.

\subsection{Analysis of the Knowledge of Medicinal Plants between} Local Experts and the General Community. Data obtained from the general community presented a higher richness of plant families and medicinal species when compared with data obtained from local experts. The difference was highly significant for total species richness $\left(\chi^{2}=11.921, P=\right.$ $0.0006)$ and exclusive species richness $\left(\chi^{2}=42.667, P=\right.$ $0.0001)$ but was not significantly different for the richness of plant families $\left(\chi^{2}=1.463, P=0.2265\right.$, Figure 3$)$. The results indicate that the knowledge of local experts managed to represent the richness of useful plant families cited by the general community but not the total number of species (Table 3). However, the most species (84.2\%) mentioned by at least 20 informants from the general community were also mentioned by local experts. This result indicates that expert informants mentioned medicinal plant species that are better known among other members of the community.

The numbers of exotic and native species mentioned by local experts and that by the general community were not significantly different according to Williams' $G$-test $(G=$ $0.9369, P=0.3331$ ). Among exclusive species, we also did not observe any significant differences between native and exotic plants $(G=0.153, P=0.6957)$, suggesting that local experts and the general community presented a similar citation repertoire of native and exotic species.

There was no significant difference between the relative importance (RI) of species mentioned by local experts and that by general community $(H=0.7899, P=0.3741)$. We observed a significant correlation between local experts and the general community in the number of indications per species according to the Spearman correlation test ( $\mathrm{rs}=$ $0.515, P<0.0001)$. This result suggests that local experts and the general community provided similar information regarding the indication of medicinal plant species. Among the ten species with higher RI mentioned by local experts and the general community, the species Borreria verticillata L. G. Mey., Hymenaea martiana Hayne, Mentha piperita L., Pithecellobium cochliocarpum (Gomez) Macbr., and Schinus terebinthifolius Raddi occurred in both studies. These results show that using local experts to provide information on the indications of medicinal plants was useful in the given context. However, the same was not observed for data on species richness. Thus, we recommend engaging the

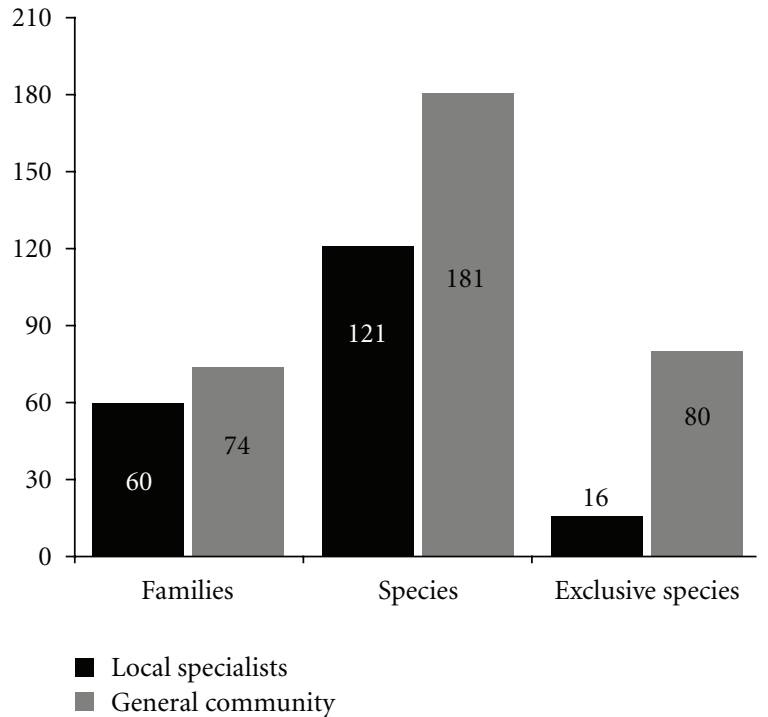

Figure 3: Comparison of the richness of families, species, and exclusive species between local experts and the general community.

whole community to gather such data. Such precaution may prevent a large number of known species from being neglected, as in our study.

\section{Discussion}

4.1. Richness of Medicinal Plants Mentioned by Informants. The study showed high diversity in the knowledge of medicinal plants, with significant results for species prescribed for basic health care. The acceptance of folk medicine and the limited access to public healthcare services in the community may be factors contributing to the knowledge of medicinal species in local medical practices.

A few plant families prominent in this study, such as Lamiaceae and Asteraceae, are reported as very diverse taxonomic groups in the literature. Their high diversity probably reflects a greater amount of bioactive compounds [29], which may explain their prominence in this study and in similar ones in other regions $[30,31]$.

Most medicinal plants used in the study area are exotic herbs that usually grow in anthropogenic areas, such as agricultural fields, gardens, and roads. Other studies performed in forest environments in different regions have shown that traditional communities select anthropogenic areas as important resource sources [32-36]. The frequent citation of herbs in the community of "Três Ladeiras" may be a consequence of the importance of this life form in anthropogenic areas, also due to the presence of strong bioactive compounds. In a study conducted in the same community, Gazzaneo et al. [15] also reported that more of the medicinal plants used by experts were herbs, collected mainly in the backyards of homes and small farms.

According to Voeks [37], weeds are often abundant in easily accessible places and rich in bioactive compounds, and as a result they are widely represented in tropical medicinal floras. 
Table 3: Exclusive species list from the work of Gazzaneo et al. [15].

\begin{tabular}{|c|c|c|c|c|}
\hline Family/scientific name & Vernacular name & Habit & Origin & RI \\
\hline \multicolumn{5}{|l|}{ Asteraceae } \\
\hline Egletes viscosa (L.) Less. & Macela & Herbs & Exotic & 0.17 \\
\hline Tagetes sp. & Cravo de defunto & Herbs & - & 0.17 \\
\hline \multicolumn{5}{|l|}{ Caesalpiniaceae } \\
\hline Senna obtusifolia (L.) H.S. Irwin \& Barnbey & Mata pasto & Herbs & Exotic & 0.17 \\
\hline Tamarindus indica L. & Tamarindo & Tree & Exotic & 0.17 \\
\hline \multicolumn{5}{|l|}{ Caprifoliaceae } \\
\hline Sambucus australis Cham. \& Schlecht & Flor de sabugo & Tree & Exotic & 0.50 \\
\hline \multicolumn{5}{|l|}{ Clusiaceae } \\
\hline Symphonia sp. & Bulandi & Herbs & - & 0.17 \\
\hline \multicolumn{5}{|l|}{ Euphorbiaceae } \\
\hline Croton sp. & Marmeleiro & Shrub & Native & 0.17 \\
\hline Euphorbia thymifoliaL. & Pé de pombo & Herbs & Native & 0.67 \\
\hline \multicolumn{5}{|l|}{ Loranthaceae } \\
\hline Phthirusa pyrifolia (H.B.K.) Eichl. & Esterco de passarinho & Herbs & Native & 0.50 \\
\hline \multicolumn{5}{|l|}{ Malvaceae } \\
\hline Malva sp. & Malva branca & Herbs & 一 & 0.17 \\
\hline \multicolumn{5}{|l|}{ Poaceae } \\
\hline Brachiaria mutica (Forsk.) Stapf & Capim de planta & Herbs & Exotic & 0.33 \\
\hline Dendrocalamus giganteus Munro & Bambu & Tree & Exotic & 0.17 \\
\hline Saccharum officinarum $\mathrm{L}$. & Cana & Herbs & Exotic & 0.17 \\
\hline \multicolumn{5}{|l|}{ Rhizophoraceae } \\
\hline Rhizophora mangle L. & Mangue & Tree & Native & 0.33 \\
\hline \multicolumn{5}{|l|}{ Rubiaceae } \\
\hline Cephaelis ipecacuanha (Brot.) A. Rich. & Papeconha & Herbs & Native & 0.50 \\
\hline \multicolumn{5}{|l|}{ Rutaceae } \\
\hline Pilocarpus sp. & Jaborandi & - & - & 0.33 \\
\hline \multicolumn{5}{|l|}{ Sapotaceae } \\
\hline Pradosia sp. & Burinhê & - & - & 0.33 \\
\hline \multicolumn{5}{|l|}{ Scrophulariaceae } \\
\hline Scoparia dulcis L. & Vassourinha & Herbs & Exotic & 0.17 \\
\hline
\end{tabular}

RI: relative importance value.

4.2. Influence of Gender and Age on the Knowledge of Medicinal Plants. Women had greater knowledge of medicinal plants when compared to men in the community studied. That result was probably due to women being the caregivers for their families, a trend also observed in other studies [5, 3840]. This scenario may also reflect the different activities performed by men and women in the community because the latter must dedicate themselves to their homes and families, which help them assimilate the knowledge they will need to keep their homes healthy at an earlier age. We should add that most species from the list of mentioned plants were exotic and herbaceous plants that are found in places women are more familiar with, such as backyards.

The study showed that young informants had less knowledge of medicinal plants when compared to older ones, which can be attributed to a lack of interest in learning and practicing such knowledge in younger generations, who are increasingly influenced by modernization. Several studies have reported similar results $[38,41-45]$. It is also important to notice that older people are more experienced and have had greater contact with plant resources and time to exchange knowledge with other informants from the region. Moreover, older people are more often affected often by various illnesses, which may help to increase their repertoire of plants and indications. In addition, they are responsible for preparing home remedies for themselves and for younger people, favoring the retention of knowledge and prompting younger individuals to use the plant resource without necessarily having knowledge of the remedy or its preparation.

4.3. Analysis of the Knowledge of Medicinal Plants between Local Experts and the General Community. This research showed that studies focused on experts can generate useful information, with a satisfactory level of reliability. But these are eminently suitable for quick diagnosis about the knowledge and use of medicinal plants in a community. That approach has the advantage of minimizing costs and time 
when collecting ethnobotanical data in the community surveyed. However, studies aiming to gather such information in greater detail should ideally engage other members of the community. That may prevent a large number of known species from being neglected. Vandebroek [46] reports that the careful selection of informants is a key task of the ethnobiologist and cannot be a simple step. The author suggests that, for a scientifically rigorous research, should be involved as many participants as possible, but if time is really a limiting factor, it is necessary to select key informants who have a high degree of knowledge about plants in the region as well as a high level of consensus with others.

Within this scenario, the association between both groups of informants is also possible [45-47]. In fact, the information gathered from key informants (experts) in those studies helped prepare semistructured forms and consensus analyses among informants. In some cases, local experts are used as facilitators for data collection, accompanying the researcher during interviews with other members of the community. These studies serve as examples of the advantages and limitations of different informant profiles and emphasize the importance of clearly establishing the goals the researcher hopes to achieve to best engage the most appropriate informants.

It should be noted, additionally, that there was a five-year interval between the data obtained from local experts and the general community. The influence of such a gap could not be measured or controlled, given the sampling design of each study and the dynamic character of human knowledge, and this limitation restricts the possibility of extrapolating the considerations discussed here. Moreover, we could not completely rule out the possibility that the so-called local expert informants have been added to the sample of the present study.

\section{Acknowledgments}

The authors thank the community of Três Ladeiras for the participation and Vanduís Valdino for fieldwork help. They also thank the contribution of the project "Sustainability of remnants of the Atlantic rainforest in Pernambuco and its implications for conservation and local development," a Brazilian-German scientific cooperation within the program "Science and Technology for the Atlantic Rainforest" funded by CNPq (590039/2006-7) and BMBF (01 LB 0203 A1). The authors' gratitude also goes to $\mathrm{CNPq}$ for the financial aid (Edital Universal) and productivity grant for research awarded to U. P. de Albuquerque and CAPES to CF. C. B. R. de Almeida.

\section{References}

[1] H. F. Leitão Filho, "Considerações sobre a florística de florestas tropicais e sub-tropicais do Brasil," IPEF, vol. 35, pp. 41-46, 1987.

[2] N. Myers, R. A. Mittermeler, C. G. Mittermeler, G. A. Fonseca, and J. Kent, "Biodiversity hotspots for conservation priorities," Nature, vol. 403, no. 6772, pp. 853-858, 2000.

[3] A. L. Peixoto, M. M. T. Rosa, and I. M. Silva, "Caracterização da Mata Atlântica," in Manual Metodológico para Estudos
Botânicos na Mata Atlântica, L. S. Sylvestre and M. M. T. Rosa, Eds., pp. 9-23, EDUR, Rio de Janeiro, Brazil, 2002.

[4] L. V. F. C. Cunha and U. P. Albuquerque, "Quantitative ethnobotany in an atlantic forest fragment of northeastern Brazil," Environmental Monitoring and Assessment, vol. 114, pp. 1-25, 2006.

[5] F. S. Silva, M. A. Ramos, N. Hanazaki, and U. P. Albuquerque, "Dynamics of traditional knowledge of medicinal plants in a rural community in the Brazilian semi-arid region," Brazilian Journal of Pharmacognosy, vol. 21, no. 3, pp. 382-391, 2011.

[6] A. H. Ladio and M. Lozada, "Nontimber forest product use in two human populations from Northwest Patagonia: a quantitative approach," Human Ecology, vol. 29, no. 4, pp. 367-380, 2001.

[7] I. Vandebroek, P. V. Damme, L. V. Puyvelde, S. Arrazola, and N. Kimped, "A comparison of traditional healers' medicinal plant knowledge in the Bolivian Andes and Amazon," Social Science and Medicine, vol. 59, no. 4, pp. 837-849, 2004.

[8] N. Hanazaki, J. Y. Tamashiro, H. F. Leitão-Filho, and A. Begossi, "Diversity of plant uses in two Caiçara communities from the Atlantic Forest coast, Brazil," Biodiversity and Conservation, vol. 9, no. 5, pp. 597-615, 2000.

[9] J. M. Monteiro, U. P. D. Albuquerque, E. M. F. Lins-Neto, E. L. D. Araújo, and E. L. C. Amorim, "Use patterns and knowledge of medicinal species among two rural communities in Brazil semi-arid northeastern region," Journal of Ethnopharmacology, vol. 105, no. 1-2, pp. 173-186, 2006.

[10] M. B. Quinlan and R. J. Quinlan, "Modernization and medicinal plant knowledge in a Caribbean horticultural village," Medical Anthropology Quarterly, vol. 21, no. 2, pp. 169-192, 2007.

[11] A. A. Ayantunde, M. Briejer, P. Hiernaux, H. M. J. Udo, and R. Tabo, "Botanical knowledge and its differentiation by age, gender and ethnicity in Southwestern Niger," Human Ecology, vol. 36, no. 6, pp. 881-889, 2008.

[12] D. Estomba, A. Ladio, and M. Lozada, "Medicinal wild plant knowledge and gathering patterns in a Mapuche community from north-western Patagonia," Journal of Ethnopharmacology, vol. 103, no. 1, pp. 109-119, 2006.

[13] C. F. C. B. R. Almeida, M. A. Ramos, E. L. C. Amorim, and U. P. Albuquerque, "A comparison of knowledge about medicinal plants for three rural communities in the semi-arid region of northeast of Brazil," Journal of Ethnopharmacology, vol. 127, no. 3, pp. 674-684, 2010.

[14] Agência Estadual de Planejamento e Pesquisa de Pernambuco (FIDEM/CODEPE), Diagnóstico das Reservas Ecológicas: Região Metropolitana do Grande Recife, Recife, Brazil, 2007.

[15] L. R. S. Gazzaneo, R. F. P. Lucena, and U. P. Albuquerque, "Knowledge and use of medicinal plants by local specialists in an region of Atlantic Forest in the state of Pernambuco (Northeastern Brazil)," Journal of Ethnobiology and Ethnomedicine, vol. 1, article 9, 2005.

[16] Fundação de Desenvolvimento Municipal do Interior de Pernambuco (FIAM), Perfil Municipal de Igarassu, Pernambuco, Brazil, 1997.

[17] Agência Estadual de Planejamento e Pesquisa de Pernambuco (FIDEM/CODEPE), Igarassu, Recife, Brazil, 2007.

[18] Secretaria de Ciência, Tecnologia e Meio Ambiente (SECTAM), Diagnósticos das Reservas Ecológicas da Região Metropolitana do Recife, Recife, Brazil, 2001.

[19] Agência Estadual de Planejamento e Pesquisa de Pernambuco (FIDEM/CODEPE), Monitoramento das Reservas Ecológicas da Região Metropolitana do Grande Recife, Recife, Brazil, 1993. 
[20] Instituto Brasileiro de Geografia e Estatística (IBGE), Resultados da amostra do censo demográfico, 2008, http://www .ibge.gov.br.

[21] U. P. Albuquerque, R. F. P. Lucena, and N. L. Alencar, "Métodos e técnicas para a coleta de dados etnobiológicos," in Métodos e Técnicas na Pesquisa Etnobiológica e Etnoecológica, U. P. Albuquerque, R. F. P. Lucena, and L. V. F. C. Cunha, Eds., pp. 39-64, NUPEEA, Recife, Brazil, 2010.

[22] Ministério da Saúde, Conselho Nacional de Saúde, Manual Operacional para comitês de ética em pesquisa, Ministério da Saúde/Série CNS Cadernos Técnicos, 2002.

[23] A. Davis and J. R. Wagner, "Who knows? On the importance of identifying "experts" when researching local ecological knowledge," Human Ecology, vol. 31, no. 3, pp. 463-489, 2003.

[24] K. Bailey, Methods of Social Research, The Free Press, New York, NY, USA, 4th edition, 1994.

[25] World Health Organization (WHO), International Statistical Classification of Diseases and Related Health Problems, 10th revision, 2006.

[26] U. P. Albuquerque, P. M. Medeiros, A. L. S. Almeida et al., "Medicinal plants of the caatinga (semi-arid) vegetation of NE Brazil: a quantitative approach," Journal of Ethnopharmacology, vol. 114, no. 3, pp. 325-354, 2007.

[27] B. C. Bennett and G. T. Prance, "Introduced plants in the indigenous pharmacopoeia of northern South America," Economic Botany, vol. 54, no. 1, pp. 90-102, 2000.

[28] M. Ayres and M. Ayres-Júnior, BioEstat 5.0-Aplicações estatísticas nas áreas das ciências bio-médicas, Universidade Federal do Pará, Pará, Brazil, 2007.

[29] M. Giday, Z. Asfaw, and Z. Woldu, "Ethnomedicinal study of plants used by Sheko ethnic group of Ethiopia," Journal of Ethnopharmacology, vol. 132, no. 1, pp. 75-85, 2010.

[30] M. Tadesse, D. Hunde, and Y. Getachew, "Survey of medicinal plants used to treat human diseases in Seka Cherkosa, Jimma Zone, Ethiopia," Ethiopian Journal of Health Sciences, vol. 15, pp. 89-106, 2005.

[31] M. Giday, Z. Asfaw, and Z. Woldu, "Medicinal plants of the Meinit ethnic group of Ethiopia: an ethnobotanical study," Journal of Ethnopharmacology, vol. 124, no. 3, pp. 513-521, 2009.

[32] R. A. Voeks, "Tropical forest healers and habitat preference," Economic Botany, vol. 50, no. 4, pp. 381-400, 1996.

[33] M. C. M. Amorozo, "Uso e diversidade de plantas medicinais em Santo Antônio de Leverger, MT, Brasil," Acta Botanica Brasílica, vol. 16, pp. 189-203, 2002.

[34] M. Giday, T. Teklehaymanot, A. Animut, and Y. Mekonnen, "Medicinal plants of the Shinasha, Agew-Awi and Amhara peoples in northwest Ethiopia," Journal of Ethnopharmacology, vol. 110, no. 3, pp. 516-525, 2007.

[35] J. R. S. Tabuti, S. S. Dhillion, and K. A. Lye, "Firewood use in Bulamogi County, Uganda: species selection, harvesting and consumption patterns," Biomass and Bioenergy, vol. 25, no. 6 , pp. 581-596, 2003.

[36] S. K. Uniyal, K. N. Singh, P. Jamwal, and B. Lal, "Traditional use of medicinal plants among the tribal communities of Chhota Bhangal, Western Himalaya," Journal of Ethnobiology and Ethnomedicine, vol. 2, article 14, 2006.

[37] R. A. Voeks, "Disturbance pharmacopoeias: medicine and myth from the humid tropics," Annals of the Association of American Geographers, vol. 94, no. 4, pp. 868-888, 2004.

[38] A. Begossi, N. Hanazaki, and J. Y. Tamashiro, "Medicinal plants in the Atlantic Forest (Brazil): knowledge, use, and conservation," Human Ecology, vol. 30, no. 3, pp. 281-299, 2002.
[39] S. Collins, X. Martins, A. Mitchell, A. Teshome, and J. T. Arnason, "Quantitative ethnobotany of two East Timorese cultures," Economic Botany, vol. 60, no. 4, pp. 347-361, 2006.

[40] T. Teklehaymanot, "Ethnobotanical study of knowledge and medicinal plants use by the people in Dek Island in Ethiopia," Journal of Ethnopharmacology, vol. 124, no. 1, pp. 69-78, 2009.

[41] N. I. Hilgert, "Plants used in home medicine in the Zenta River basin, Northwest Argentina," Journal of Ethnopharmacology, vol. 76, no. 1, pp. 11-34, 2001.

[42] H. Fassil, We do what we know: local health knowledge and home-based medicinal plant use in Ethiopia, Ph.D. thesis, Green College, Oxford University, 2003.

[43] T. Gedif and H. J. Hahn, "The use of medicinal plants in selfcare in rural central Ethiopia," Journal of Ethnopharmacology, vol. 87, no. 2-3, pp. 155-161, 2003.

[44] D. Hunde, Z. Asfaw, and E. Kelbessa, "Use and management of ethnoveterinary medicinal plants by indigenous people in "Boosat", Welenchiti area," Ethiopian Journal of Biological Sciences, vol. 3, pp. 113-132, 2004.

[45] K. Srithi, H. Balslevb, P. Wangpakapattanawonga, P. Srisangac, and C. Trisonthia, "Medicinal plant knowledge and its erosion among the Mien (Yao) in northern Thailand," Journal of Ethnopharmacology, vol. 123, no. 2, pp. 335-342, 2009.

[46] I. Vandebroek, "The Dual Intracultural and Intercultural Relationship between Medicinal Plant Knowledge and Consensus," Economic Botany, vol. 64, no. 4, pp. 303-317, 2010.

[47] S. L. Cartaxo, M. M. A. Souza, and U. P. Albuquerque, "Medicinal plants with bioprospecting potential used in semiarid northeastern Brazil," Journal of Ethnopharmacology, vol. 131, no. 2, pp. 326-342, 2010. 


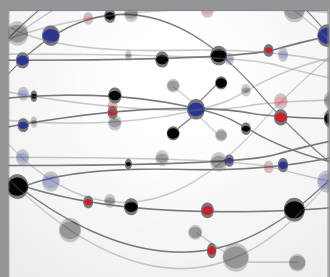

The Scientific World Journal
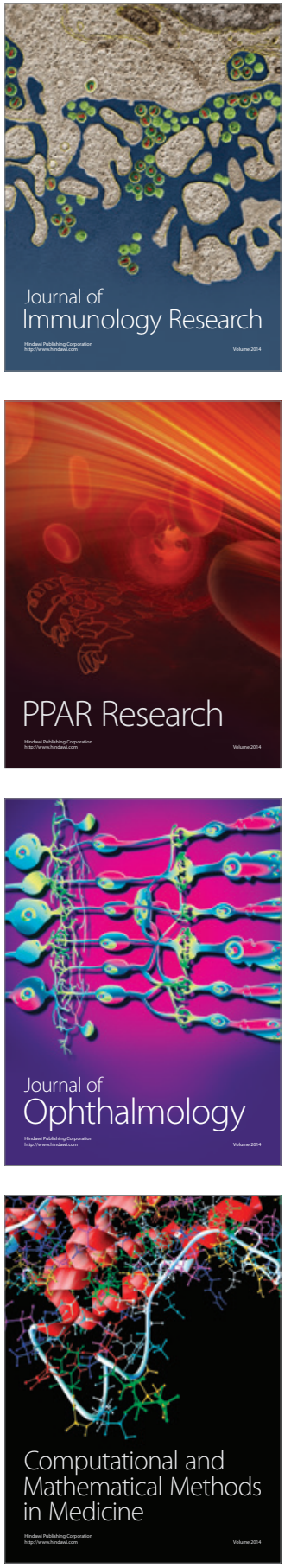

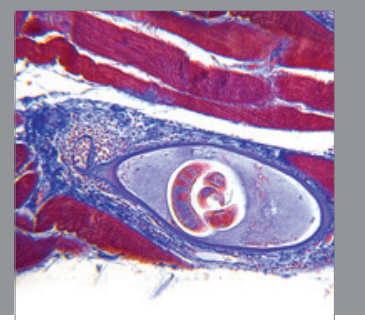

Gastroenterology

Research and Practice
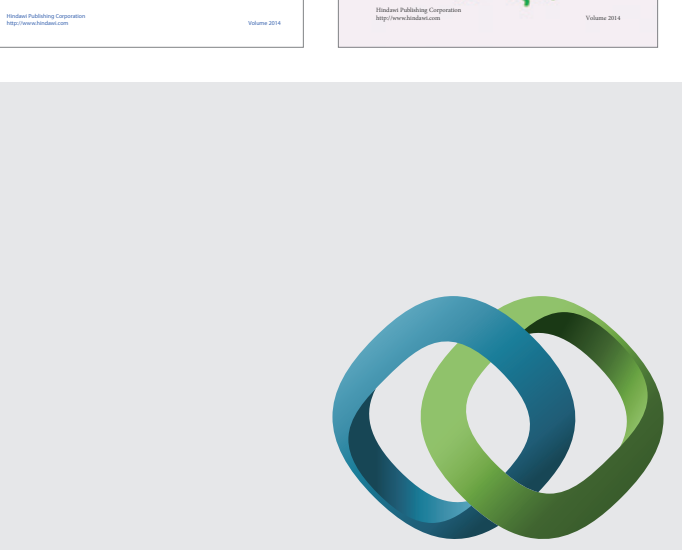

\section{Hindawi}

Submit your manuscripts at

http://www.hindawi.com
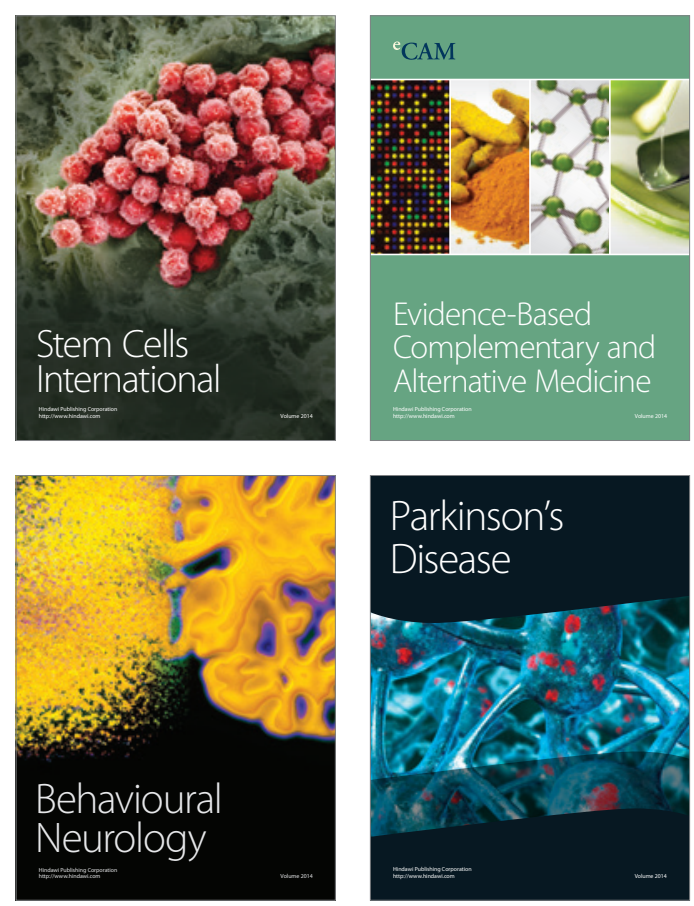

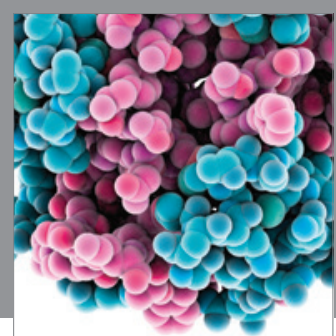

Journal of
Diabetes Research

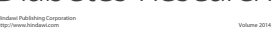

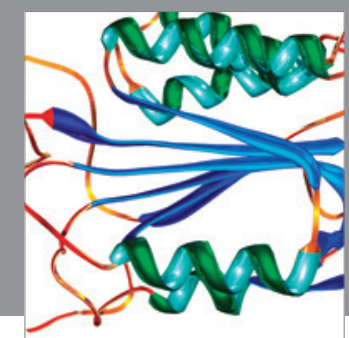

Disease Markers
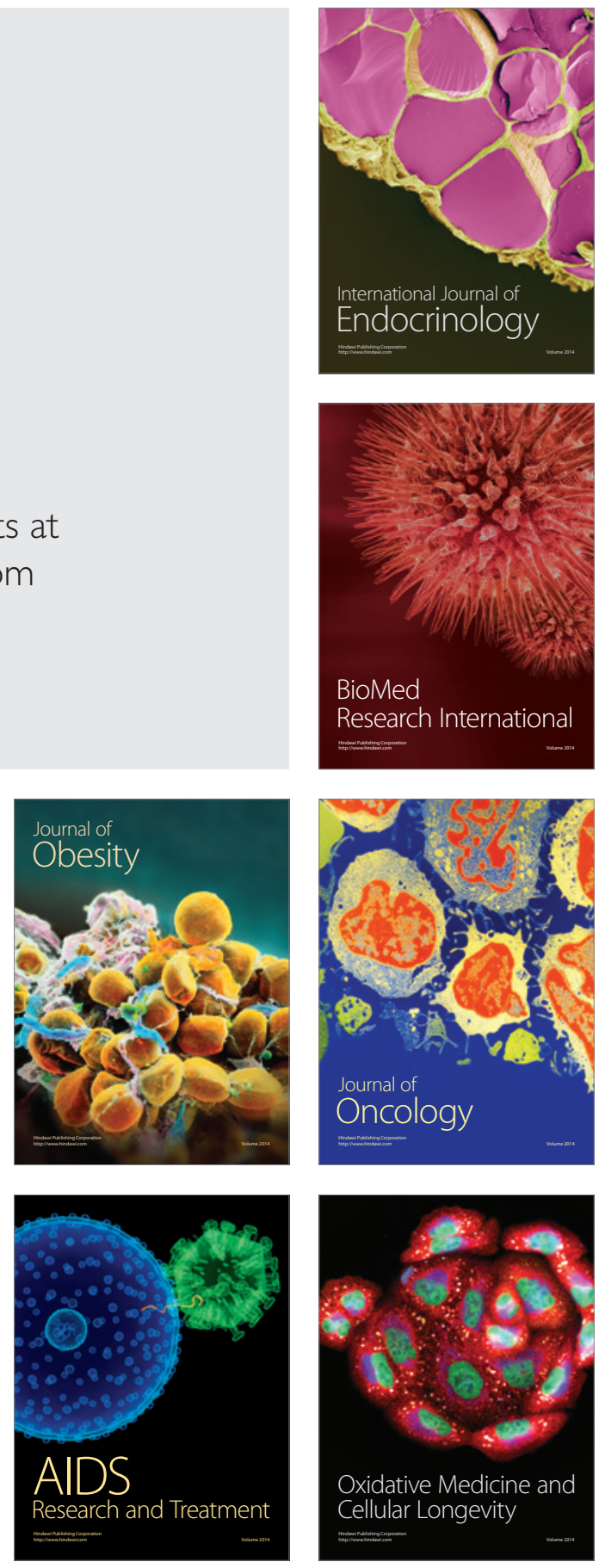